\title{
MULTIPLIERS ON DUAL $\boldsymbol{A}^{*}$-ALGEBRAS
}

\author{
B. J. TOMIUK
}

\begin{abstract}
Let $A$ be an $A^{*}$-algebra which is a dense *-ideal of a $B^{*}$-algebra $\mathfrak{A}$. We use tensor products and the algebra $M_{l}(A)$ of left multipliers on $A$ to obtain a characterization of duality in $A$. We show, moreover, that if $A$ is dual then $M_{l}(A)$ is algebra isomorphic to the second conjugate space $\mathfrak{A}^{* *}$ of $\mathfrak{A}$ when $\mathfrak{I}^{* *}$ is given Arens product.
\end{abstract}

1. Introduction. Let $A$ be an $A^{*}$-algebra which is a dense *-ideal of a $B^{*}$-algebra $A$. In [8] a necessaary and sufficient condition was given for $A$ to be dual which was expressed in terms of the weak operator topology on $M_{r}(A)$, the algebra of right multipliers on $A$, and a certain property of $A$ called property (P2). In this paper we give several characterizations of property (P2) and then use some of them to give conditions for duality in $A$. Our presentation here differs somewhat from that in [8]. We use the tensor product approach as developed in [3] and [6]. Particularly in $\$ 2$ we follow closely the presentation given in [3].

We shall use the notation of [8]. An $A^{*}$-algebra $A$ is said to be of the first kind if it is an ideal of its completion $\mathfrak{A}$ in the auxiliary norm $|\cdot|$. It follows that there exists a constant $k>0$ such that $\|x y\| \leqslant k\|x\||y|$ for all $x \in A, y$ $\in \mathfrak{U}\left[4\right.$, Lemma 4, p. 18]. If $A$ is a modular annihilator $A^{*}$-algebra then $|\cdot|$ is unique $[1,(1.3)$, p. 6] so that $\mathfrak{A}$ is also unique.

2. The property (P2). Let $A$ be a Banach algebra, $A^{*}$ and $A^{* *}$ its first and second conjugate spaces. Let $A \hat{\otimes} A^{*}$ be the projective tensor product of $A$ and $A^{*}$ [7, pp. 92-95]. Then $A \hat{\otimes} A^{*}$ is a Banach space with elements of the form $\sum_{k=1}^{\infty} a_{k} \otimes f_{k}$ such that $\sum_{k=1}^{\infty}\left\|a_{k}\right\|\left\|f_{k}\right\|<\infty, a_{k} \in A, f_{k} \in A^{*}$, and the norm given by

$$
\|h\|=\inf \left\{\sum_{k=1}^{\infty}\left\|a_{k}\right\|\left\|f_{k}\right\|: h=\sum_{k=1}^{\infty} a_{k} \hat{\otimes} f_{k}\right\} .
$$

For $a \in A, f \in A^{*}$, let $a f \in A^{*}$ be given by (af) $x=f(x a), x \in A$. This makes $A^{*}$ into a left Banach $A$-module. We note that if $\sum_{k=1}^{\infty} a_{k} \hat{\otimes} f_{k} \in A \hat{\otimes}$ $A^{*}$, then $\sum_{k=1}^{\infty} a_{k} f_{k} \in A^{*}$. Let $\psi$ be the continuous linear map of $A \hat{\otimes} A^{*}$ into $A^{*}$ given by

Received by the editors June 17, 1976.

AMS (MOS) subject classifications (1970). Primary 46K10, 46L20; Secondary 47B05.

Key words and phrases. Dual $A^{*}$-algebra, multipliers, Arens product, projective tensor product, Banach $\boldsymbol{A}$-module.

() American Mathematical Society 1977 


$$
\psi(a \hat{\otimes} f)=a f \quad\left(a \in A, f \in A^{*}\right) .
$$

For $a \in A, F \in A^{* *}$, let $F a \in A^{* *}$ be given by $(F a) f=F(a f), f \in A^{*}$. Then $A^{* *}$ is a right Banach $A$-module. (See [6] for the definition and properties of Banach $A$-modules.)

Let $A \circ A^{*}$ be the Banach space $A \hat{\otimes} A^{*} / \operatorname{ker}(\psi)$ with the usual quotient norm, where $\operatorname{ker}(\psi)$ is the kernel of $\psi$. Then $\left(A \circ A^{*}\right)^{*}$ consists of all those $\mathscr{F} \in\left(A \hat{\otimes} A^{*}\right)^{*}$ which vanish on $\operatorname{ker}(\psi)$. Now, for each $F \in A^{* *}$, let $\mathscr{F}_{F} \in$ $\left(A \hat{\otimes} A^{*}\right)^{*}$ be given by

$$
\mathscr{F}_{F}(a \hat{\otimes} f)=F(\psi(a \hat{\otimes} f))=F(a f) \quad\left(a \in A, f \in A^{*}\right)
$$

Each $\mathscr{F}_{F}$ vanishes on $\operatorname{ker}(\psi)$, so that $\left\{\mathscr{F}_{F}: F \in A^{* *}\right\}$ may be indentified as a subspace of $\left(A \circ A^{*}\right)^{*}$. Moreover if $F a=0$ for all $a \in A$ implies $F=0$, then $F \rightarrow \mathscr{F}_{F}$ is a one-to-one map of $A^{* *}$ into $\left(A \hat{\otimes} A^{*}\right)^{*}$. Thus in this case $F \rightarrow \mathscr{F}_{F}$ identifies $A^{* *}$ as a subspace of $\left(A \circ A^{*}\right)^{*}$.

Let $\sigma$ denote the $w^{*}$-topology of $\left(A \hat{\otimes} A^{*}\right)^{*}$.

LEMMA 2.1. $\left(A \circ A^{*}\right)^{*}$ is the $\sigma$-closure of $\left\{\mathscr{F}_{F}: F \in A^{* *}\right\}$.

Proof. We have

$$
\begin{aligned}
\operatorname{ker}(\psi) & =\left\{\sum_{k=1}^{\infty} a_{k} \hat{\otimes} f_{k} \in A \hat{\otimes} A^{*}: \sum_{k=1}^{\infty} a_{k} f_{k}=0\right\} \\
& =\left\{\sum_{k=1}^{\infty} a_{k} \hat{\otimes} f_{k} \in A \hat{\otimes} A^{*}: F\left(\sum_{k=1}^{\infty} a_{k} f_{k}\right)=0, F \in A^{* *}\right\} .
\end{aligned}
$$

Thus $\operatorname{ker}(\psi)=\bigcap\left[\operatorname{ker}\left(\mathscr{F}_{F}\right): F \in A^{* *}\right]$, which means that $\operatorname{ker}(\psi)$ is the polar of $\left\{\mathscr{F}_{F}: F \in A^{* *}\right\}$. Therefore, by the Bipolar Theorem [7, p. 126], $\left(A \circ A^{*}\right)^{*}$ is the $\sigma$-closure of $\left\{\mathscr{F}_{F}: F \in A^{* *}\right\}$. This completes the proof.

We observe that if $A^{2}=(0)$, then $\operatorname{ker}\left(\mathscr{F}_{F}\right)=A \hat{\otimes} A^{*}$, for every $F \in A^{* *}$, so that $\operatorname{ker}(\psi)=A \hat{\otimes} A^{*}$ and consequently $\left(A \circ A^{*}\right)^{*}=(0)$.

Let $\mathscr{B}\left(A, A^{* *}\right)$ be the Banach space of all bounded linear operators $T$ : $A \rightarrow A^{* *}$ normed with the operator bound norm. For each $\mathscr{F} \in\left(A \hat{\otimes} A^{*}\right)^{*}$, let $T_{\mathscr{F}}$ be the map on $A$ into $A^{* *}$ given by

$$
\left(f, T_{\mathscr{F}}(a)\right)=\mathscr{F}(a \hat{\otimes} f) \quad\left(a \in A, f \in A^{*}\right) .
$$

Then clearly $T_{\mathscr{F}} \in \mathscr{B}\left(A, A^{* *}\right)$ for every $\mathscr{F} \in\left(A \hat{\otimes} A^{*}\right)^{*}$, and it is easy to check that the map $\phi: \mathscr{F} \rightarrow T_{\mathscr{F}}$ is an isometric isomorphism of $\left(A \hat{\otimes} A^{*}\right)^{*}$ onto $\mathfrak{B}\left(A, A^{* *}\right)$. Give $\mathfrak{B}\left(A, A^{* *}\right)$ the image of the $\sigma$ topology by the map $\phi$. Following Máté [3], we shall refer to this topology as the ultraweak topology on $\Re\left(A, A^{* *}\right)$.

Now consider $A^{* *}$ as a right Banach $A$-module and let $\operatorname{Hom}_{A}\left(A, A^{* *}\right)$ be the set of all $T \in \mathscr{B}\left(A, A^{* *}\right)$ such that $T(a b)=T(a) b, a, b \in A$. The canonical map $\pi: A \rightarrow A^{* *}$ belongs to $\Re\left(A, A^{* *}\right)$ since $\pi(a b) f=f(a b)=$ $\pi(a)(b f)$ for all $a, b \in A$ and $f \in A^{*}$. For each $F \in A^{* *}$, let $T_{F}: A \rightarrow A^{* *}$ be given by $T_{F}(a)=F a, a \in A$. Then $T_{F} \in \operatorname{Hom}_{A}\left(A, A^{* *}\right)$, and we have $\phi\left(\mathscr{F}_{F}\right)$ 
$=T_{F}$ for all $F \in A^{* *}$. In view of Lemma 2.1 and the fact that the ultraweak closure of $\left\{T_{F}: F \in A^{* *}\right\} \subseteq \operatorname{Hom}_{A}\left(A, A^{* *}\right)$ we have

LEMMA 2.2. $\phi\left(\left(A \circ A^{*}\right)^{*}\right) \subseteq \operatorname{Hom}_{A}\left(A, A^{* *}\right)$ and is the ultraweak closure of $\left\{T_{F}: F \in A^{* *}\right\}$.

If $\phi$ maps $\left(A \circ A^{*}\right)^{*}$ onto $\operatorname{Hom}_{A}\left(A, A^{* *}\right)$, we shall write $\left(A \circ A^{*}\right)^{*} \cong$ $\operatorname{Hom}_{A}\left(A, A^{* *}\right)$. In this case, for every $T \in \operatorname{Hom}_{A}\left(A, A^{* *}\right), \mathcal{F}_{T} \in\left(A \hat{\otimes} A^{*}\right)^{*}$, given by $\mathscr{F}_{T}(a \hat{\otimes} f)=(f, T(a))$, belongs to $\left(A \circ A^{*}\right)^{*}$. In particular,

$$
\mathscr{F}_{\pi}(a \hat{\otimes} f)=f(a), \quad \text { for all } a \in a, f \in A^{*} \text {. }
$$

We recall that a Banach algebra $A$ is said to have property (P2) if: $a_{k} \in A, f_{k} \in A^{*}, \quad \sum_{k=1}^{\infty}\left\|a_{k}\right\|\left\|f_{k}\right\|<\infty$ and $\sum_{k=1}^{\infty} a_{k} f_{k}=0$ implies that $\sum_{k=1}^{\infty} f_{k}\left(a_{k}\right)=0$. (This is the left-hand version of the definition given in [8].)

TheOREM 2.3. Let $A$ be a Banach algebra. Then the following statements are equivalent:

(i) $A$ has property (P2).

(ii) For $h=\sum_{k=1}^{\infty} a_{k} \hat{\otimes} f_{k} \in \operatorname{ker}(\psi)$ we have $\sum_{k=1}^{\infty} f_{k}\left(a_{k}\right)=0$.

(iii) $\mathscr{F}_{\pi}$ vanishes on $\operatorname{ker}(\psi)$.

(iv) $\mathscr{F}_{\pi} \in\left(A \circ A^{*}\right)^{*}$.

(v) $\operatorname{Hom}_{A}\left(A, A^{* *}\right) \cong\left(A \circ A^{*}\right)^{*}$.

(vi) There exists a net $\left\{u_{\alpha}\right\}$ in $A$ such that $\left\{\mathscr{F}_{\pi\left(u_{\alpha}\right)}\right\}$ converges to $\mathscr{F}_{\pi}$ in the $w^{*}$-topology on $\left(A \hat{\otimes} A^{*}\right)^{*}$.

Proof. (i) $\Leftrightarrow$ (ii) and (iii) $\Leftrightarrow$ (iv) are clear.

(iv) $\Rightarrow$ (vi). Suppose (iv) holds. Then $\operatorname{ker}\left(\mathscr{F}_{\pi}\right) \supset \operatorname{ker}(\psi)$. We have

$$
\mathscr{F}_{\pi}(h)=\mathscr{F}_{\pi}\left(\sum_{k=1}^{\infty} a_{k} \hat{\otimes} f_{k}\right)=\sum_{k=1}^{\infty} \mathscr{F}_{\pi}\left(a_{k} \hat{\otimes} f_{k}\right)=\sum_{k=1}^{\infty} f_{k}\left(a_{k}\right),
$$

for all $h=\sum_{k=1}^{\infty} a_{k} \hat{\otimes} f_{k} \in A \hat{\otimes} A^{*}$. Since $\left\{\mathscr{F}_{F}: F \in A^{* *}\right\}$ is $\sigma$-dense in $\left(A \circ A^{*}\right)^{*}$, there exists a net $\left\{F_{\alpha}\right\}$ in $A^{* *}$ such that $\mathscr{F}_{F_{\alpha}}(h) \rightarrow \mathscr{F}_{\pi}(h)$ for all $h \in A \hat{\otimes} A^{*}$. Since $\pi(A)$ is $w^{*}$-dense in $A^{* *}$ and $\sigma$ is weaker than the $w^{*}$-topology on $A^{* *}$, it follows that $\left\{\mathscr{F}_{\pi(a)}: a \in A\right\}$ is $\sigma$-dense in $\left(A \circ A^{*}\right)^{*}$. Hence there exists a net $\left\{u_{\alpha}\right\}$ in $A$ such that $\left\{\mathscr{F}_{\pi\left(u_{a}\right)}\right\} \sigma$-converges to $\mathscr{F}_{\pi}$. We have

$$
\begin{aligned}
\mathscr{F}_{\pi\left(u_{\alpha}\right)}(h) & =\pi\left(u_{\alpha}\right)(\psi(h))=\sum_{k=1}^{\infty} \pi\left(u_{\alpha}\right)\left(a_{k} f_{k}\right) \\
& =\left(\sum_{k=1}^{\infty} a_{k} f_{k}\right)\left(u_{\alpha}\right)=\sum_{k=1}^{\infty}\left(a_{k} f_{k}\right)\left(u_{\alpha}\right) \\
& =\sum_{k=1}^{\infty} f_{k}\left(u_{\alpha} a_{k}\right) .
\end{aligned}
$$

Thus 


$$
\lim _{\alpha} \mathscr{F}_{\pi\left(u_{\alpha}\right)}(h)=\lim _{\alpha} \sum_{k=1}^{\infty} f_{k}\left(u_{\alpha} a_{k}\right)=\sum_{k=1}^{\infty} f_{k}\left(a_{k}\right)=\mathscr{F}_{\pi}(h),
$$

for all $h=\sum_{k=1}^{\infty} a_{k} \hat{\otimes} f_{k} \in A \hat{\otimes} A^{*}$.

(vi) $\Rightarrow(\mathrm{v})$. We have $\phi\left(\left(A \circ A^{*}\right)^{*}\right) \subseteq \operatorname{Hom}_{A}\left(A, A^{* *}\right)$. We need only show that $\operatorname{Hom}_{A}\left(A, A^{* *}\right) \subseteq \phi\left(\left(A \circ A^{*}\right)^{*}\right)$. Let $T \in \operatorname{Hom}_{A}\left(A, A^{* *}\right)$ and let $\mathscr{F}_{T}$ be the corresponding element of $\left(A \hat{\otimes} A^{*}\right)^{*}$. Then, using (1), we obtain (identifying $A$ as a subset of $A^{* *}$ and $A^{*}$ as a subset of $\left.A^{* * *}\right)$ :

$$
\begin{aligned}
\mathscr{F}_{T}\left(\sum_{k=1}^{\infty} a_{k} \hat{\otimes} f_{k}\right) & =\sum_{k=1}^{\infty} \mathscr{F}_{T}\left(a_{k} \hat{\otimes} f_{k}\right)=\sum_{k=1}^{\infty}\left(f_{k}, T\left(a_{k}\right)\right) \\
& =\sum_{k=1}^{\infty}\left(T^{*} f_{k}\right)\left(a_{k}\right)=\lim _{\alpha} \sum_{k=1}^{\infty}\left(T^{*} f_{k}\right)\left(u_{\alpha} a_{k}\right) \\
& =\lim _{\alpha} \sum_{k=1}^{\infty} f_{k}\left(T\left(u_{\alpha} a_{k}\right)\right)=\lim _{\alpha} \sum_{k=1}^{\infty} f_{k}\left(T\left(u_{\alpha}\right) a_{k}\right) \\
& =\lim _{\alpha} \sum_{k=1}^{\infty}\left(T^{*} a_{k} f_{k}\right)\left(u_{\alpha}\right)=\sum_{k=1}^{\infty} T^{*}\left(a_{k} f_{k}\right),
\end{aligned}
$$

where $T^{*}$ is the conjugate of $T$. Hence if $\sum_{k=1}^{\infty} a_{k} f_{k}=0$ then $\mathscr{F}_{T}\left(\sum_{k=1}^{\infty} a_{k} \hat{\otimes} f_{k}\right)$ $=0$, so that $\operatorname{ker}(\psi) \subset \operatorname{ker}\left(\mathscr{F}_{T}\right)$. Thus $\operatorname{Hom}_{A}\left(A, A^{* *}\right) \subseteq \phi\left(\left(A \circ A^{*}\right)^{*}\right)$ and so $\operatorname{Hom}_{A}\left(A, A^{* *}\right) \cong\left(A \circ A^{*}\right)^{*}$.

(v) $\Rightarrow$ (iv). This is clear since $\pi \in \operatorname{Hom}_{A}\left(A, A^{* *}\right)$.

3. Dual $A^{*}$-algebras. Let $A$ be a Banach algebra. A map $T: A \rightarrow A$ is called a left (resp. right) multiplier if $T(a b)=T(a) b$ (resp. $T(a b)=a T(b)$ ), for all $a, b \in A$. Let $M_{l}(A)$ (resp. $M_{r}(A)$ ) be the set of all bounded linear left (resp. right) multipliers on $A . M_{l}(A)$ and $M_{r}(A)$ are Banach algebras under the usual operations for operators and the operator bound norm. We observe that if $T \in M_{l}(A)$ then the composite map $\pi \circ T \in \operatorname{Hom}_{A}\left(A, A^{* *}\right)$. Let $\phi_{\pi}$ be the map of $M_{l}(A)$ into $\operatorname{Hom}_{A}\left(A, A^{* *}\right)$ given by

$$
\phi_{\pi}(T)=\pi \circ T \quad\left(T \in M_{l}(A)\right) .
$$

For any Banach space $X$, let $\delta(X)$ denote the closed unit ball of $X$. It follows from the proof of [8, Theorem 4.7, p. 286] that if $A$ is a dual $A^{*}$-algebra of the first kind then $\delta\left(M_{l}(A)\right)$ is $\tau_{l}$-compact, where $\tau_{l}$ is the weak operator topology on $M_{l}(A)$. (We take the left-hand version of the arguments in $[8$, p. 286].)

TheOREM 3.1. Let $A$ be an $A^{*}$-algebra of the first kind. Then the following statements are equivalent:

(i) $A$ is dual.

(ii) $\phi_{\pi}\left(M_{l}(A)\right)$ is the ultraweak closure of $\left\{T_{F}: F \in A^{* *}\right\}$.

Proof. (i) $\Rightarrow$ (ii). Suppose $A$ is dual. Then, by [8, Theorem 4.7, p. 286], it has property (P2) and therefore, by Theorem 2.3, $\left(A \circ A^{*}\right)^{*} \cong$ $\operatorname{Hom}_{A}\left(A, A^{* *}\right)$. Hence $\operatorname{Hom}_{A}\left(A, A^{* *}\right)$ is the ultraweak closure of $\left\{T_{F}: F \in\right.$ 
$\left.A^{* *}\right\}$. Now $T_{F}(a) f=(F a) f=(F * \pi(a)) f$ and, by [9, Theorem 5.2, p. 830], $\pi(A)$ is an ideal of $A^{* *}$ when $A^{* *}$ is given either Arens product, so that $T_{F}(a) \in \pi(A)$ for all $a \in A$. Therefore $T_{F}=\pi \circ T$, for some $T \in M_{l}(A)$, and so $\left\{T_{F}: F \in A^{* *}\right\} \subseteq \phi_{\pi}\left(M_{l}(A)\right)$. Let $Q \in \operatorname{Hom}_{A}\left(A, A^{* *}\right)$. Then, by Lemma 2.2 and the fact that $\left(A \circ A^{*}\right)^{*} \cong \operatorname{Hom}_{A}\left(A, A^{* *}\right)$, there exists a net $\left\{F_{\alpha}\right\}$ in $A^{* *}$ such that $\left(f, T_{F_{\alpha}}(a)\right) \rightarrow(f, Q(a))$ for all $a \in A, f \in A^{*}$. Let $T_{\alpha} \in M_{l}(A)$ be such that $T_{F_{\alpha}}=\pi \circ T_{\alpha}$, for all $\alpha$. Then $\left(f, T_{F_{\alpha}}(a)\right)=f\left(T_{\alpha}(a)\right)$ since $T_{\alpha}(a) \in A$. But, by [8, Theorem 4.7, p. 286], $M_{l}(A)$ is $\tau_{l}$-complete. Hence there exists $T \in M_{l}(A)$ such that $f\left(T_{\alpha}(a)\right) \rightarrow f(T(a))$, for all $a \in A, f$ $\in A^{*}$. This shows that $\pi(T(a)) f=(f, Q(a))$, for all $a \in A, f \in A^{*}$, or equivalently, $\pi(T(a))=Q(a)$, for all $a \in A$, i.e., $Q=\pi \circ T$. Thus $Q \in$ $\phi_{\pi}\left(M_{l}(A)\right)$ and so $\operatorname{Hom}_{A}\left(A, A^{* *}\right)=\phi_{\pi}\left(M_{l}(A)\right)$. Since $\left(A \circ A^{*}\right)^{*} \cong$ $\operatorname{Hom}_{A}\left(A, A^{* *}\right)$ and since $\left(A \circ A^{*}\right)^{*}$ is the $\sigma$-closure of $\left\{\mathscr{F}_{F}: F \in A^{* *}\right\}$, it follows that $\phi_{\pi}\left(M_{l}(A)\right)$ is the ultraweak closure of $\left\{T_{F}: F \in A^{* *}\right\}$.

(ii) $\Rightarrow$ (i). Suppose (ii) holds. Then, in view of Lemma 2.2, $M_{l}(A)$ is isometrically isomorphic to $\left(A \circ A^{*}\right)^{*}$. From Lemma 2.1 we obtain $\delta\left(\left(A \circ A^{*}\right)^{*}\right)$ is $\sigma$-compact, and since $\tau_{l}$ is weaker than the ultraweak topology on $M_{l}(A)$, it follows that $\mathcal{S}\left(M_{l}(A)\right)$ is $\tau_{l}$-compact and therefore $\tau_{l}$-complete. Let $I$ be the identity element of $M_{l}(A)$. Since $\left\{T_{F}: F \in A^{* *}\right\}$ is ultraweak dense in $\phi_{\pi}\left(M_{l}(A)\right)$, there exists a net $\left\{F_{\alpha}\right\}$ in $A^{* *}$ such that $T_{F_{\alpha}}$ converges ultraweakly to $\pi \circ I=\pi$, or equivalently, $\mathscr{F}_{F_{\alpha}} \sigma$-converges to $\mathscr{F}_{\pi}$. Since $\pi(A)$ is $w^{*}$-dense in $A^{* *}$ and the $w^{*}$-topology is stronger than the $\sigma$-topology on $A^{* *}$, it follows that there exists a net $\left\{u_{\alpha}\right\}$ in $A$ such that $\left\{\mathscr{F}_{\pi\left(u_{a}\right)}\right\} \sigma$-converges to $\mathscr{F}_{\pi}$ and so, by Theorem $2.3, A$ has property (P2). Therefore, by [8, Theorem 4.7, p. 287], $A$ is dual.

Corollary 3.2. Let $A$ be an $A^{*}$-algebra of the first kind. Then $A$ is dual if and only if $\phi_{\pi}\left(M_{l}(A)\right)=\phi\left(\left(A \circ A^{*}\right)^{*}\right)$.

COROllaRy 3.3. Let $A$ be a modular annihilator $A^{*}$-algebra of the first kind. If $\operatorname{Hom}_{A}\left(A, A^{* *}\right)$ is the ultraweak closure of $\left\{T_{F}: F \in A^{* *}\right\}$ then $A$ is dual.

Proof. By [9, Theorem 5.2, p. 830], $\pi(A)$ is an ideal of $A^{* *}$ so that $T_{F}$ maps $A$ into $\pi(A)$ for every $F \in A^{* *}$. Hence if $\operatorname{Hom}_{A}\left(A, A^{* *}\right)$ is the ultraweak closure of $\left\{T_{F}: F \in A^{* *}\right\}$, then $\operatorname{Hom}_{A}\left(A, A^{* *}\right)=\phi_{\pi}\left(M_{l}(A)\right)$ by the proof above. Therefore $A$ is dual by Theorem 3.1.

\section{A realization of the algebra $M_{l}(A)$.}

TheOREM 4.1. Let $A$ be a dual $A^{*}$-algebra of the first kind and let $\mathfrak{A}$ be its completion. Let $\pi_{\mathfrak{A}}$ be the canonical map of $\mathfrak{A}$ into $\mathfrak{A}^{* *}$. Then $\pi_{\mathfrak{X}}(A)$ is an ideal of $\mathfrak{U}^{* *}$ when $\mathfrak{U}^{* *}$ is given Arens product.

Proof. Let $x \in A, F \in \mathfrak{U}^{* *}$ and let $\left\{e_{\alpha}\right\}$ be a maximal orthogonal family of selfadjoint minimal idempotents in $A$. By [4, Theorem 16, p. 30], $\Sigma_{\alpha} e_{\alpha} x$ is summable to $x$ in the norm $\|\cdot\|$, and hence there exists only a countable number of $e_{\alpha}$ for which $e_{\alpha} x \neq 0$, say $e_{\alpha_{1}}, e_{\alpha_{2}}, \ldots$ Since $A$ and $\mathfrak{A}$ have the 
same socle and $\pi_{\mathscr{A}}(\mathfrak{U})$ is an ideal of $\mathfrak{U}^{* *}$, it follows that $F * \pi_{\mathfrak{Y}}\left(e_{\alpha_{i}}\right) \in \pi_{\mathfrak{Y}}(A)$ for $i=1,2, \ldots$ Let $m, n$ be positive integers, $m \leqslant n$. By [4, Lemma 4, p. 18 ], we have

$$
\begin{aligned}
& \left\|\sum_{i=1}^{n} F * \pi_{\mathfrak{A}}\left(e_{\alpha_{i}}\right) \pi_{\mathfrak{A}}(x)-\sum_{i=1}^{m} F * \pi_{\mathfrak{A}}\left(e_{\alpha_{i}}\right) * \pi_{\mathfrak{A}}(x)\right\| \\
& =\left\|\left(\sum_{i=m+1}^{n} F * \pi_{\mathfrak{A}}\left(e_{\alpha_{i}}\right)\right)\left(\sum_{i=m+1}^{n} \pi_{\mathfrak{A}}\left(e_{\alpha_{i}}\right) * \pi_{\mathfrak{Y}}(x)\right)\right\| \\
& \leqslant k\left\|\left(\sum_{i=m+1}^{n} F * \pi_{\mathfrak{X}}\left(e_{\alpha_{i}}\right)\right)\left(\sum_{i=m+1}^{n} \pi_{\mathfrak{M}}\left(e_{\alpha_{i}}\right) * \pi_{\mathfrak{Y}}(x)\right)\right\| \\
& \leqslant k|F|\left\|\sum_{i=m+1}^{n} \pi_{\mathfrak{A}}\left(e_{\alpha_{i}}\right)\right\|\left\|\sum_{i=m+1}^{n} \pi_{\mathfrak{A}}\left(e_{\alpha_{i}}\right) * \pi_{\mathfrak{A}}(x)\right\| \\
& \leqslant k|F|\left\|\sum_{i=m+1}^{n} \pi_{\mathfrak{A}}\left(e_{\alpha_{i}}\right) * \pi_{\mathscr{U}}(x)\right\|,
\end{aligned}
$$

where $|F|$ denotes the norm of $F$ in $\mathfrak{U}^{* *}$ and $k$ is a positive constant. Thus $\left\{\sum_{i=1}^{n} F * \pi_{\mathfrak{A}}\left(e_{\alpha_{i}}\right) * \pi_{\mathscr{U}}(x)\right\}$ is a Cauchy sequence in $\pi_{\mathfrak{X}}(A)$ with respect to the norm $\|\cdot\|$, and so there exists $z \in A$ such that $\pi_{A}(z)=$ $\sum_{i=1}^{\infty} F * \pi_{\mathfrak{Y}}\left(e_{\alpha_{i}}\right) * \pi_{\mathscr{Y}}(x)$. Since $\sum_{i=1}^{\infty} F * \pi_{\mathfrak{A}}\left(e_{\alpha_{i}}\right) * \pi_{\mathscr{A}}(x)$ also converges to $\pi_{\mathfrak{X}}(z)$ and to $F * \pi_{\mathscr{A}}(x)$ in the norm $|\cdot|$, we have $\pi_{\mathscr{Y}}(z)=F * \pi_{\mathfrak{X}}(x)$.Hence $F * \pi_{\mathscr{A}}(x)$ $\in \pi_{\mathfrak{Y}}(A)$, for all $x \in A$ and $F \in \mathfrak{U}^{* *}$. Similarly we can show that $\pi_{\mathfrak{X}}(x) * F$ $\in \mathfrak{U}^{* *}$, for all $x \in A$ and $F \in \mathfrak{U}^{* *}$. Therefore $\pi_{\mathfrak{A}}(A)$ is an ideal of $\mathfrak{U}^{* *}$.

Theorem 4.2. Let $A$ be a dual $A^{*}$-algebra of the first kind and $\mathfrak{A}$ its completion. Then $M_{l}(A)$ is algebra isomorphic to $\mathfrak{U}^{* *}$ when $\mathfrak{U}^{* *}$ is given Arens product. This isomorphism is given by the following relation: For each $T \in$ $M_{l}(A)$ there exists a unique $F_{T} \in \mathfrak{U}^{* *}$ such that

$$
\pi_{\mathfrak{A}}(T x)=F_{T} * \pi_{\mathfrak{A}}(x) \quad(x \in A) .
$$

Proof. For each $x \in A$, let $\|x\|_{A}^{\prime}=\sup \{\|x y\|:\|y\| \leqslant 1, y \in A\}$. Then $\|\cdot\|_{A}^{\prime}$ is a norm on $A$ which is equivalent to $|\cdot|[4$, Theorem 18, p. 31]. Hence if $T \in M_{l}(A)$ and $x \in A$, then

$$
\begin{aligned}
\|T x\|_{A}^{\prime} & =\sup \{\|T(x) y\|:\|y\| \leqslant 1, y \in A\} \\
& =\sup \{\|T(x y)\|:\|y\| \leqslant 1, y \in A\} \\
& \leqslant\|T\| \sup \{\|x y\|:\|y\| \leqslant 1, y \in A\} \\
& \leqslant k^{\prime}\|T\||x|,
\end{aligned}
$$

where $k^{\prime}$ is a constant $>0$. Thus $|T x| \leqslant k^{\prime \prime}|x|$ for all $x \in A$ and some constant $k^{\prime \prime}>0$. Since $A$ is dense in $\mathfrak{A}$, it follows that $T$ has a unique bounded extension $T^{\prime}$ to $\mathfrak{A}$. Clearly $T^{\prime} \in M_{l}(\mathfrak{U})$. By [2, Corollary 3.2, p. 509], there exists a unique $F_{T} \in \mathfrak{A}^{* *}$ such that $\pi_{\mathfrak{Q}}(T x)=F_{T} * \pi_{\mathfrak{Q}}(x)$ for all $x \in A$. 
Since, by Theorem 4.1, $\pi_{\mathfrak{Y}}(A)$ is an ideal of $\mathfrak{U}^{* *}$, we have that $T \rightarrow F_{T}$ is an algebra isomorphism of $M_{l}(A)$ onto $\mathfrak{A}^{* *}$.

\section{REFERENCES}

1. B. A. Barnes, Subalgebras of modular annihilator algebras, Proc. Cambridge Philos. Soc. 66 (1969), 5-12. MR 41 \#4236.

2. B. D. Malviya and B. J. Tomiuk, Multiplier operators on $B^{*}$-algebras, Proc. Amer. Math. Soc. 31 (1972), 505-510. MR 46 \# 4215.

3. L. Máté, On representation of module-homomorphisms (multipliers), Studia Sci. Math. Hungar. 8 (1973), 187-192. MR 51 \# 1286.

4. T. Ogasawara and K. Yoshinaga, Weakly completely continuous Banach*-algebras, J. Sci. Hiroshima Univ. Ser. A 18 (1954), 15-36. MR 16, 1126.

5. C. E. Rickart, General theory of Banach algebras, Van Nostrand, Princeton, N.J., 1960. MR 22 \#5903.

6. M. Rieffel, Induced Banach representations of Banach algebras and locally compact groups, J. Functional Analysis 1 (1967), 443-491. MR 36 \#6544.

7. H. H. Schaefer, Topological vector spaces, Macmillan, New York, 1966. MR 33 \# 1689.

8. B. J. Tomiuk, Multipliers and duality in $A^{*}$-algebras, Proc. Amer. Math. Soc. 50 (1975), 281-288. MR 51 \#8834.

9. Pak-ken Wong, Modular annihilator $A^{*}$-algebras, Pacific J. Math. 37 (1971), 825-834. MR 46 \#4231.

Department of Mathematics, University of Ottawa, Ottawa, Ontario, Canada K1N 6N5 